Original study

\title{
Outcome in patients with diabetes mellitus undergoing isolated surgical aortic valve replacement
}

\author{
Alina Cristina Iliescu ${ }^{1,2}$, Mariana Floria ${ }^{*}, 2,3$, Mihaela Grecu ${ }^{1}$, Ionuț Achiței ${ }^{1}$, Cristina \\ Luca $^{1}$, Grigore Tinică ${ }^{1,2}$
}

1"Prof. Dr. George I.M. Georgescu" Institute of Cardiovascular Diseases lasi, Romania; ""Grigore T. Popa" University of Medicine and Pharmacy, lasi, Romania; ${ }^{3} 3^{\text {rd }}$ Internal Medicine Department, "Sf. Spiridon" University Emergency Hospital lasi, Romania

\begin{abstract}
Background: The risk scoring systems used in cardiac surgery (including EuroSCORE II) include only insulin-dependent diabetes. Diabetes mellitus (DM) is a marker of poor prognosis after surgical myocardial revascularization, but its impact in patients with isolated surgical aortic valve replacement (SAVR) has not been well established. Aim: We aimed to analyze differences in outcomes and surgical risk in patients with and without type 2 DM (T2DM), which underwent SAVR. Material and methods: We included retrospectively the patients hospitalized for SAVR between January 2000 and June 2014 in Cardiovascular Surgery Unit of Cardiovascular Diseases Institute. Preoperative parameters and early postoperative outcome in patients with and without T2DM were compared. Results: A number of 1191 patients were included $(65.4 \pm 13$ years; $67.8 \%$ men); 144 (12.07\%) out of those were with T2DM. Biological prostheses were performed in $22 \%$ of patients. Mean age was higher in T2DM group $(p=0.005)$. The mean EuroSCORE II risk score was $5.49 \pm 0.63$ in T2DM and 4.89 \pm 0.17 in non-T2DM patients $(p=0.579)$. In these 2 groups, preoperative left ventricular systolic function was comparable. Mean values of cardiopulmonary bypass time were $137.88 \pm 5.71$ minutes in T2DM, $149.48 \pm 4.8$ minutes in non-T2DM patients $(p=0.714)$. The need of inotropic therapy was an important predictor for postoperative evolution ( $6 \pm 2$ days in nonT2DM and $15 \pm 4$ days in T2DM; $p=0.008$ ). Conclusions: Patients with T2DM undergoing SAVR have a nonsignificantly higher operative risk score comparing with non-T2DM patients. In spite of this, T2DM seems to be a risk factor that could worsen the postoperative outcome, by requiring prolonged inotropic treatment.
\end{abstract}

Keywords: diabetes; aortic stenosis; aortic surgery; surgical risk

\section{Introduction}

Diabetes mellitus (DM) represents the fourth cause of death worldwide according International Diabetes Federation, requiring increased health resource [1]. Patients with type 2 DM (T2DM) should be considered as being at high cardiovascular risk if older than 40 years, even in the absence of coronary risk factors or preexisting cardiovascular disease [1]. Diabetes mellitus is a common risk factor

Received: October 2017; Accepted after review: February 2018; Published: March 2018.

${ }^{*}$ Corresponding author: Mariana Floria, MD, PhD,

FESC, "Grigore T. Popa" University of Medicine and Pharmacy, 16 University Street, laşi, România.

Phone: +40.232.301.600 Fax: +40.232.211.820

E-mail: floria mariana@yahoo.com for vascular atherosclerosis and aortic valve calcification, the initial process that leads to aortic stenosis [2, 3]. According to the current guidelines, the etiology of calcific severe aortic stenosis is now dominated by atherosclerotic degeneration in patients $>65$ years in Europe and North America [4].

Patients with DM and calcific severe aortic stenosis have multiple cardiac risk factors. Diabetes and aortic stenosis are both associated with left ventricular hypertrophy and increased left ventricular mass, concomitant with altered diastolic function [5, 6]. Approximately two third of patients with aortic calcific disease had a history of multiple cardiovascular risk factors like hypercholesterolemia, hypertension or coronary artery disease [7, 8]. Despite of 
normal LDL-C plasma level due to the important heterogeneity in the aortic stenosis progression rate and prognosis [9].

The risk scoring systems used in cardiac surgery (including EuroSCORE II) include only insulin-dependent diabetes as an important risk factor to predict 30-day mortality [9]. Diabetes mellitus is considered a risk factor of poor outcomes after cardiac surgery in certain cardiovascular diseases (i.e. coronary artery disease); however its impact in patients with isolated (without coronary arteries lesions which need surgical revascularization or mitral valve surgery) surgical aortic valve replacement (SAVR) has not been well established.

The aim of this study was to analyze differences in outcome and surgical risk in patients with and without T2DM, which underwent isolated SAVR.

\section{Material and methods}

We included retrospectively patients admitted in Cardiovascular Surgery Unit of Cardiovascular Diseases Institute between January 2000 and June 2014 for SAVR. We analyzed differences in outcome and surgical risk in patients with and without T2DM, which underwent isolated SAVR.

The inclusion criteria were patients over 18 years old, referred to our center for isolated SAVR who signed the informed consent.

The exclusion criteria were patients who also needed an aorto-coronary bypass or mitral surgery; patients who refused to be included in this study.

Patients were divided into two groups according T2DM presence (T2DM versus nonT2DM). All data obtained from previous medical recordings were recorded into an extensive database.

Preoperative parameters (dyslipidemia, smoking, arterial hypertension, glomerular filtration rate, preoperative arrhythmias, cerebrovascular disease and transthoracic echocardiography data) and early postoperative outcome until their discharge (inotropic medication need, re-intervention, stroke $>72 \mathrm{~h}$, coma $>24 \mathrm{~h}$, mediastinitis, treatment with statins, it seems to be still an sepsis, multiple system organ failure, death) were compared.

EuroSCORE II score was calculated using the online available calculators (http://www.EuroSCORE.org/calc.html; http://209.220.160.181/STSWebRiskCalc261) to assess the individual risk of postoperative death.

Patients were fully informed about the nature of the study and provided written informed consent which was approved by the local Ethics Committee.

\section{Statistical analysis}

Statistical results are given as numbers and percentages for categorical variables, and as mean \pm standard deviation for continuous variables. We used Kolmogorov-Smirnov test for assessing normality of distribution of quantitative variables; these variables were described by median and percentiles or by mean and standard deviation. We also used Fisher's exact test and chi-square test for comparing the frequencies of nominal variables. With T-test or Mann-Whitney test we analyzed the differences in the means and medians or between groups. With Spearman coefficient we assessed the non-normal distribution of the data. A $p$ value of $<0.05$ was considered statistically significant. Data were analyzed using SPSS 17.0 (SPSS inc., Chicago, IL, USA) statistical software package and Microsoft Office Excel 2007.

\section{Results}

This study included 1191 patients who underwent SAVR; 144 (12.07\%) were with T2DM patients and 26 patients (18.05\%) out of those were with insulin-dependent T2DM. Mean age was $65.4 \pm 13$ years, and $67.8 \%$ were men. The biological prostheses were performed in $22 \%$ of patients, $28.47 \%$ of T2DM patients and $21.39 \%$ of patients in the non-T2DM group ( $\mathrm{p}=0.087$ ).

Preoperative parameters of patients included in the study, comparing T2DM and non-T2DM patients are presented in Table 1. 
Table 1. Preoperative data of patients included in the study

\begin{tabular}{|c|c|c|c|}
\hline Parameter & $\begin{array}{l}\text { T2DM Patients } \\
(\mathrm{N}=144)\end{array}$ & $\begin{array}{c}\text { Non-T2DM Patients } \\
(\mathrm{N}=1047)\end{array}$ & P Value \\
\hline Age (mean \pm SD) & $69.8 \pm 8.4$ & $64.8 \pm 13.6$ & 0.005 \\
\hline Men (\%) & 54 & 58 & 0.540 \\
\hline BMI (kg/m2) & $27 \pm 6$ & $25 \pm 8$ & 0.723 \\
\hline Arterial hypertension (\%) & 39.6 & 25.6 & 0.002 \\
\hline Dyslipidemia (\%) & 12.32 & 87.67 & 0.03 \\
\hline Smoking (\%) & 36.1 & 37.4 & 0.8 \\
\hline Family history of CVD (\%) & 50.7 & 38.9 & 0.057 \\
\hline Cerebrovascular Disease (\%) & 11.1 & 10.5 & 0.2 \\
\hline GFR $<60 \mathrm{cc} / \mathrm{min} / 1.73 \mathrm{~m} 2$ & 40.98 & 59.02 & 0.007 \\
\hline Preoperative arrhythmias (\%) & 3.6 & 31.8 & 0.089 \\
\hline LVEF $<30 \%$ (number of patients/\%) & $5(3.47 \%)$ & $73(6.97 \%)$ & 0.038 \\
\hline LVEF $=30-50 \%$ (number of patients $/ \%$ ) & $81(56.25 \%)$ & $610(58.26 \%)$ & 0.744 \\
\hline LVEF> $50 \%$ ( number of patients/\%) & $58(40.27 \%)$ & $364(34.76 \%)$ & 0.476 \\
\hline PAPs (mmHg) & 47 & 43 & 0.09 \\
\hline
\end{tabular}

Mean age was significantly higher in T2DM group $(p=0.005)$. Family history of cardiovascular disease was more frequent in T2DM group $(p=0.057)$, as well as arterial hypertension $(p=0.002)$. Renal function was significantly impaired in T2DM group $(p=0.007)$.

Preoperative left ventricular systolic function showed no significant differences between groups, irrespective of dysfunction degree $(p=0.744)$.

Mean EuroSCORE II risk score in T2DM versus non-T2DM patients was $5.49 \pm 0.63$ and $4.89 \pm 0.17(p=0.579)$.
Cardiopulmonary bypass time in T2DM patients was shorter $(137.88 \pm 5.71$ minutes versus $149.48 \pm 4.80$ minutes; $p=0.714$ ).

Postoperative outcomes were analyzed in Table 2. There were no statistically significant differences between T2DM and non-T2DM group, except for inotropic medication need (15 \pm 4 days versus $6 \pm 2$ days; $p=0.008$ ). Hospitalization duration were similar for T2DM and non-T2DM group (21.40 \pm 9.60 days versus $21.71 \pm 9.70$ days; $p=0.63)$.

Table 2. Postoperative data in T2DM versus non-T2DM patients

\begin{tabular}{lccc}
\hline \multicolumn{1}{c}{ Parameter } & $\begin{array}{c}\text { T2DM Patients } \\
(\mathbf{N = 1 4 4 )}\end{array}$ & $\begin{array}{c}\text { Non-T2DM Patients } \\
(\mathbf{N = 1 0 4 7 )}\end{array}$ & P Value \\
\hline Increased inotropic medication need (days) & $15 \pm 4$ & $6 \pm 2$ & $\mathbf{0 . 0 0 8}$ \\
\hline Re-intervention (\%) & 10.55 & 7.2 & 0.206 \\
\hline Stroke >72 h (\%) & 1.25 & 4.8 & 0.127 \\
\hline Coma >24h (\%) & 6.32 & 6.66 & 0.572 \\
\hline Mediastinitis (\%) & 1.33 & 0.20 & 0.248 \\
\hline Sepsis (\%) & 1.53 & 2.83 & 0.175 \\
\hline Multiple System Organ Failure (\%) & 3.33 & 4.41 & 0.514 \\
\hline Death (\%) & 0.6 & 0.57 & 0.78 \\
\hline
\end{tabular}




\section{Study Limitation}

Our study has several limitations, being a single center, non-randomized, retrospective analysis and thus exposed to biases. We tried to temper the bias by including a larger lot of patients, to achieve statistical significance. In addition a longer follow-up would be of interest. Studies with a longer follow-up to evaluate the long-term outcomes need to be done.

\section{Discussion}

It is well known that patients with T2DM have a high cardiovascular risk. Diabetes is a common risk factor encountered in patients undergoing SAVR; the prevalence of both T2DM and aortic disease is increasing.

Another strong risk factor for cardiovascular diseases regardless of the presence of T2DM is the age. In our study, age was significantly higher in T2DM group. Studies including large diabetic groups have shown that age is an important predictor for cardiovascular events and advanced atherosclerosis, but little is known about the absolute risk, age-related in diabetic patients $[10,11]$.

The percentage of dyslipidemia was higher in non-T2DM group, probable because of more aggressive risk-reduction therapies with intensive lipid lowering agents in T2DM group.

Hypertension was significantly associated with T2DM; this is well known in literature. Recent data suggests that hypertensive patients are more predisposed to T2DM than those normotensive [12].

Among T2DM complications, chronic kidney disease is the most important; leading important costs in the medical system. Our study confirms that renal dysfunction is significantly present in patients with T2DM. Renal impairment is included in EuroSCORE II [9]. In this study the patients with T2DM have had a lower creatinine clearance; despite this the EuroSCORE II risk score was not significantly different in the two groups.

Female gender is a risk factor included in EuroSCORE II [8]. Even it is known that women could have worse outcome regarding in-hospital mortality after SAVR [13], in our study no significant difference regarding gender was observed.

Patients with T2DM did not have a longer cardiopulmonary bypass time. No differences in postoperative complications (including the infectious complications) were unregistered in both groups (T2DM versus non-T2DM). However, patients with T2DM have required a longer period of inotropic support. Inotropic medication is known as the strongest independent predictor for long-term mortality in patients with SAVR [14]. In this study no significant differences in deaths were unregistered at 30 days after SAVR, in patients with T2DM versus non-T2DM.

Preoperative risk stratification is an important step in choosing the adequate management but needs to be reset after the surgery, depending on intraoperative and early postoperative factors, as the need for inotropes. A major complication following cardiac surgery with cardiopulmonary bypass is the low cardiac output syndrome, which affects up to $20 \%$ of patients and represents a major challenge, as it is associated with increased morbidity and mortality [15]. Diabetes mellitus and preoperative renal dysfunction combined increase the risk of low cardiac output syndrome by $50 \%$ [16].

Inotropic stimulation affects both systole and diastole and may enhance diastolic dysfunction even the patient has a normal ejection fraction. The need of positive inotropic drugs is a marker of severity after cardiac surgery, because patients who require vasopressors are at higher risk than those who do not [17].

However, a very recently published article about patients with diabetes and elevated cardiovascular risk concluded that: „even after extensive adjustment for underlying disease burden, there was a persistent association for low diastolic blood pressure with subclinical myocardial injury and risk of myocardial infarction, [18]. Because DM is a heterogeneous disease with significant variability in the glucose control strategies (which could determine variable degree of long term complications), and because the postoperative outcome could depend on the 
type of treatment but also on preoperative glycemic control, these patients with high cardiovascular risk seem to be a heterogeneous population with variable postoperative outcomes even in the absence of myocardial revascularization need.

\section{Conclusion}

Patients with T2DM undergoing SAVR have a non-significantly higher operative risk

\section{References}

1. Aschner P. New IDF clinical practice recommendations for managing type 2 diabetes in primary care. Diabetes Res Clin Pract 2017; 132:169-170.

2. Katz R, Wong ND, Kronmal R, et al. Features of the metabolic syndrome and diabetes mellitus as predictors of aortic valve calcification in the multi-ethnic study of atherosclerosis. Circulation 2006; 113(17):2113-2119.

3. Otto CM, Bonow RO. Valvular Heart Disease. In Bonow RO, Mann DL, Zipes DP, Libby $P$, Braunwald $E$ (eds) Braunwald's Heart Disease. $9^{\text {th }}$ edition. Philadelphia: Elsevier, 2012; 1468-1539.

4. Vahanian $A$, Alfieri $O$, Andreotti $F$, et al. Guidelines on the management of valvular heart disease (version 2012). Joint Task Force on the Management of Valvular Heart Disease of the European Society of Cardiology (ESC); European Association for Cardio-Thoracic Surgery (EACTS). Eur Heart J 2012; 33(19):2451-2496.

5. Grossman W, Jones D, McLaurin LP. Wall stress and patterns of hypertrophy in the human left ventricle. J Clin Invest 1975; 56(1):56-64.

6. Tadic M, llic S, Cuspidi C, et al. Left ventricular mechanics in untreated normotensive patients with type 2 diabetes mellitus: a two- and three-dimensional speckle tracking study. Echocardiography 2015; 32(6):947-955.

7. Grundy SM, Cleeman JI, Daniels SR, et al. Diagnosis and Management of the Metabolic Syndrome: An American Heart score comparing with non-T2DM patients. In spite of this, T2DM patients require prolonged inotropic treatment. Therefore, type $2 \mathrm{DM}$ might be a risk factor that could worsen the postoperative outcome.

\section{Conflict of interest}

The authors declare that they have no competing interests.

Association/National Heart, Lung, and Blood Institute Scientific Statement. Circulation 2005; 112(17):2735-2752.

8. Mathieu P, Després JP, Pibarot P. The valvulo-metabolic risk in calcific aortic valve disease. Can J Cardiol 2007; 23(Suppl B):32B-39B.

9. Nashef $S A$, Roques $F$, Sharples LD, et al. EuroSCORE II. Eur J Cardiothorac Surg 2012; 41(4):734-744.

10. Stevens RJ, Kothari V, Adler Al, Stratton IM; United Kingdom Prospective Diabetes Study (UKPDS) Group. The UKPDS risk engine: a model for the risk of coronary heart disease in Type II diabetes (UKPDS 56). Clin Sci (Lond) 2002; 101(6):671-679.

11. Booth GL, Kapral MK, Fung K, Tu JV. Relation between age and cardiovascular disease in men and women with diabetes compared with non-diabetic people: a population-based retrospective cohort study. Lancet 2006; 368(9529):29-36.

12. Taskinen MR, Boren J. New insights into the pathophysiology of dyslipidemia in type 2 diabetes. Atherosclerosis 2015; 239(2):483-495.

13. Chaker Z, Badhwar V, Alqahtani F, et al. Sex differences in the utilization and outcomes of surgical aortic valve replacement for severe aortic stenosis. J Am Heart Assoc 2017; 6(9), pii:e006370. doi: 10.1161/JAHA.117.006370.

14. Lehmann $S$, Meyer $A$, Schroeter $T$, et al. Midterm durability and hemodynamic performance of a third-generation bovine pericardial prosthetic aortic valve: the 
Leipzig experience. Ann Thorac Surg 2017; 103(6):1933-1939.

15. St André AC, DelRossi A. Hemodynamic management of patients in the first 24 hours after cardiac surgery. Crit Care Med 2005; 33(9):2082-2093.

16. Pan W, Hindler K, Lee VV, Vaughn WK, Collard CD. Obesity in diabetic patients undergoing coronary artery bypass graft surgery is associated with increased postoperative morbidity. Anesthesiology 2006; 104(3):441-447.
17. Butterworth $\mathrm{JF} 4^{\text {th }}$, Legault $\mathrm{C}$, Royster RL, Hammon JW Jr. Factors that predict the use of positive inotropic drug support after cardiac valve surgery. Anesth Analg 1998; 86(3):461-467.

18. Bergmark BA, Scirica BM, Steg PG, et al; SAVOR-TIMI 53 Investigators. Blood pressure and cardiovascular outcomes in patients with diabetes and high cardiovascular risk. Eur Heart J 2018; doi: 10.1093/eurheartj/ehx809. 